\title{
含二氮杂慕砌块化合物的荧光性能构效关系研究及应用
}

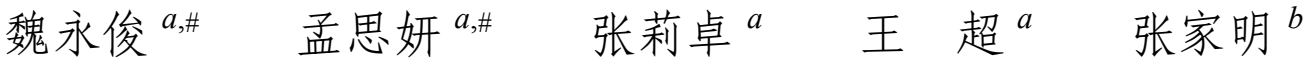 \\ 宛瑜*,c 吴 翚*, $, a, c$ \\ ( ${ }^{a}$ 江苏师范大学化学化工学院 江苏省功能材料绿色合成重点实验室 徐州 221116) \\ ( ${ }^{b}$ 江苏省泗洪县教育局 泗洪 223900) \\ ( ${ }^{c}$ 江苏药用植物生物技术重点实验室 徐州 221116)
}

\begin{abstract}
摘要 通过文献方法合成了具有较好平面共轭结构和较高电子云密度的 5-氨基-2-芳基-3H-色烯 $[4,3,2-d e][1,6]$ 并二氮杂 萗-4-腈(4)和 5-氨基-2-芳基-3H-喹啉 $[4,3,2-d e][1,6]$ 并二氮杂菜-4-腈(6), 研究了稠合环数、苂光助色团数量以及取代基类 型对产物发光性能的影响，探索了其结构与发光性能之间的关系. 在发光性能构效关系指导下初步探究了化合物 4 对 9 种金属离子的识别，并从产物中笁选得到了具有进一步研究价值的、具开发为有机苂光材料潜质的产物.
\end{abstract}

关键词 二氮杂菱; 苂光性能; 构效关系; 应用

\section{Study and Application of the Structure-Fluorescence Relationship of Naphthyridine-Containing Compounds}

\author{
Wei, Yongjun $^{a, \#} \quad$ Meng, Siyan $^{a, \#} \quad$ Zhang, Lizhuo $^{a} \quad$ Wang, Chao $^{a} \quad$ Zhang, Jiaming $^{b}$ \\ Wan, $\mathrm{Yu}^{*, c} \mathrm{Wu}, \mathrm{Hui}^{*, a, c}$ \\ ( ${ }^{a}$ School of Chemistry and Chemical Engineering, Jiangsu Normal University, Jiangsu Key Laboratory of \\ Green Synthesis of Functional Material, Xuzhou 221116) \\ ( ${ }^{b}$ Sihong County Bureau of Education, Sihong 223900) \\ ( ${ }^{c}$ Jiangsu Key Laboratory of Biotechnology on Medical Plant, Xuzhou 221116)
}

\begin{abstract}
Amino-2-aryl-3H-chromeno[4,3,2-de][1,6]naphthyridine-4-carbonitriles and 5-amino-2-aryl-3H-quinolino[4,3,2-de] $[1,6]$ naphthyridine-4-carbonitriles were synthesized via literature method. The effect of the number of the fused ring, the amount of fluorescent auxochrome and the types of substituents on the luminescent properties of the products was investigated in detail to explore the relationships between their structure and luminescence properties. In the guidance of the structureluminescence relationship, compound $\mathbf{4}$ was used as sensor to identify nine metal ions successfully. The products with potential researching and development value as organic fluorescent material were picked up.
\end{abstract}

Keywords naphthyridine; fluorescence properties; structure-luminescence relationship; application

共轭体系的大小、分子的刚性大小、取代基的电子 性质是影响有机分子发光的三个必要条件和影响因素, 因此, 我们可以通过调控有机分子的稠环数(影响共轭 体系和分子的刚性)、取代基类型(影响共轭体系和电子 离域)、杂原子类型(影响共轭体系和电子云密度)等, 来 根据我们的需要调控有机分子的发光性质(如: 发光波 长范围、强度、亮度等 $)^{[1]}$.
而高效有机发光材料的篎选不可能通过对成千上 万的合成有机分子进行一一篮选、测定来完成, 所以有 机分子合理的结构-活性关系构建就显得尤为主要.

芳基具有长的共轭体系、大的分子刚性以及大的离 域电子云密度，在体系中引入不同的芳基既可调控有机 分子的刚性和空间位阻，又可增长共轭体系，还可提供 大的离域电子云密度，是一种优秀的、可增强分子发光

*E-mail: wuhui72@yahoo.com.cn

Received November 5, 2013; revised December 17, 2013; published online January 2, 2014.

Project supported by the Natural Science Foundation of the Jiangsu Higher Education Institutions of China (No. 10KJA430050) and the Natural Science Foundation of Jiangsu Normal University (No. 11XLA06).

江苏省高校自然科学研究重大项目(No. 10KJA430050)和江苏师范大学自然科学重点基金(No. 11XLA06)资助项目.

\#共同第一作者(Authors contributed equally to this work). 


\section{性质的取代基.}

二氮杂荎是一个重要的天然产物和药物结构单 元 $^{[1,2]}$, 本身具有多种重要的生理活性, 如抗菌 ${ }^{[3 \sim 5]}$ 、局

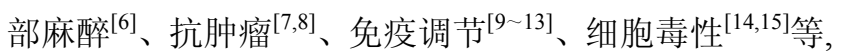
被用于治疗阿尔茨海默病 ${ }^{[16 ~ 18] ~}$ 和除草剂等 $^{[19]}$.

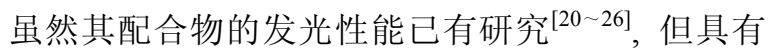
平面刚性结构和助色团的二氮杂荎本身的发光性能却 很少被系统研究. 因此, 合成不同芳基稠合和取代的二 氮杂荎化合物并对其进一步修饰, 有望获得新型光学材 料.

本论文参照文献[27]合成了具有较好平面共轭结构 和较高电子云密度的色烯 $[4,3,2-d e]$ 并 $[1,6]$ 二氮杂荟类 化合物(图 1, 产物 4)和喹啉 $[4,3,2-d e]$ 并 $[1,6]$ 二氮杂芸骨 架(图 1, 产物 6), 研究了稠合环数、荧光助色团数量以 及取代基类型对产物 6 发光性能的影响, 探索了其结构 与发光性能之间的关系. 在发光性能构效关系指导下初 步探究了化合物 4 对 9 种金属离子的识别. 从产物中篮 选得到了可被进一步研究的具有开发为有机苂光材料 潜质的产物, 为将其研发成为新型有机发光器件提供了 数据支持.
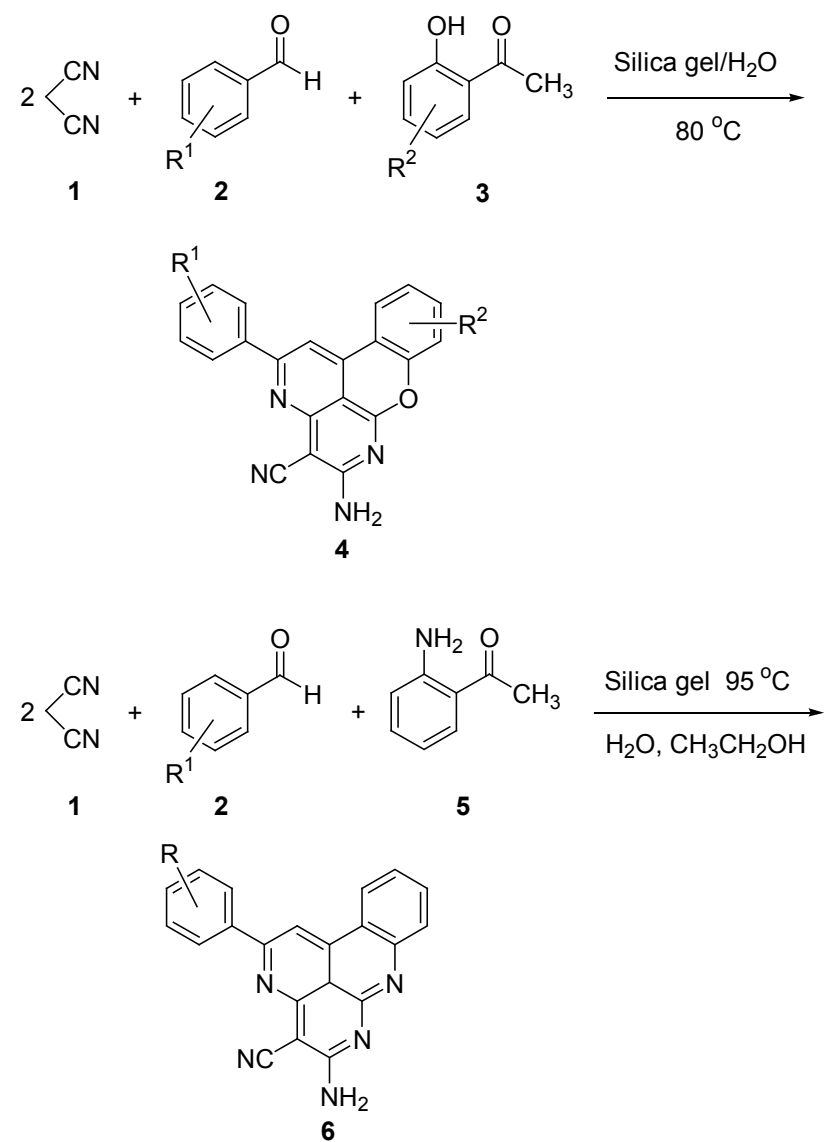

图 1 化合物 4 和 6 的合成

Figure 1 Synthesis of compounds 4 and 6

\section{1 结果与讨论}

\section{1 色烯 $[4,3,2-d e]$ 并 $[1,6]$ 二氮杂荎类化合物 4 的结} 构-苂光性能关系研究

从产物的结构可以看出：其分子具有一个大的平面 刚性共轭体系，在理论上符合发光化合物的结构要求. 实际上，该产物确实能发射很强的可裸眼识别的荧光 (图 2、图 3).

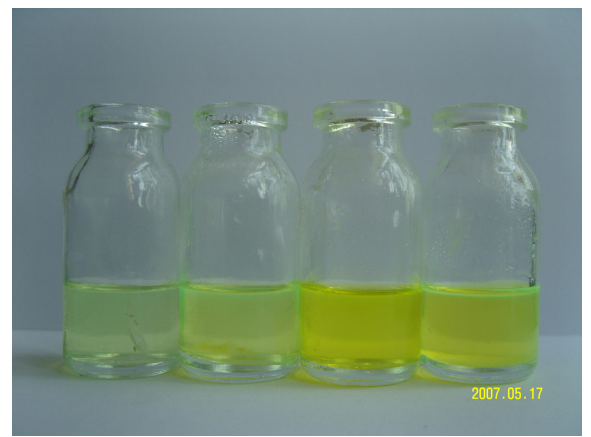

图 $2 \quad 4 a \sim 4 d$ 在日光激发下发射的荧光

Figure 2 Emission fluorescence of $\mathbf{4 a} \sim \mathbf{4 d}$ in sunlight excitation

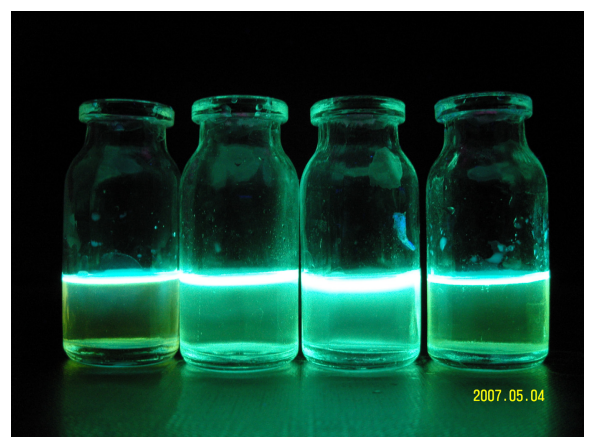

图 $34 \mathbf{a} \sim 4 \mathbf{d}$ 在 $365 \mathrm{~nm}$ 光激发下发射的苂光

Figure 3 Emission fluorescence of $\mathbf{4 a} \sim \mathbf{4 d}$ in $365 \mathrm{~nm}$ excitation

产物 4 的发光性质见表 1 和图 4, 由表 1 和图 4 可 知:

(1)所有产物都能发射较强的苂光，且与参比 9,10 二苯葱苂光的最大发射波长 $\left(\lambda_{\mathrm{em}}=432 \mathrm{~nm}\right)$ 相差不大，因 此我们选择 9,10-二苯葱作为参比物. 产物的发射波长 均在可见光范围内 $\left(\lambda_{\mathrm{em}}=453 \sim 501 \mathrm{~nm}\right)$, 所有产物的激 发波长或发射波长差别都不大，说明其发光性质主要来 自于其骨架，与取代基的电子效应、空间位阻关系不大.

(2)发射波长的大小与两个取代基的电子效应有关: $\mathrm{R}^{1}$ 为吸电子基的产物的荧光发射波长大于 $\mathrm{R}^{1}$ 为供电子 基的产物. $\mathrm{R}^{1}$ 和 $\mathrm{R}^{2}$ 具有相反的电子效应差别且越大，电 子流动有方向性和驱动力越强, 分子的极性越大, 稳定 性越高，荧光发射波长越长 $(4 \mathrm{~b}, 4 \mathrm{I}$ 和 $\mathbf{4 s})$.

(3)所有产物的 Stokes 位移均较大(147 203 nm), 
发射光受激发光影响小, 不易发生自吸收现象, 能量损 失少, 发光寿命长. 其规律同产物的荧光发射波长大小 规律, 即 $\mathrm{R}^{1}$ 为吸电子基的产物的 Stokes 位移大于 $\mathrm{R}^{1}$ 为 供电子基的产物的 Stokes 位移.

(4)产物的荧光量子产率为 $0.144 \sim 0.716$, 其中化合 物 $4 a, 4 j, 4 x$ 和 $4 z$ 的量子产率超过了参比物, 具有进一 步研究的价值. 量子产率主要决定于分子的电子云密 度, 产物量子产率的变化规律与 $\lambda_{\mathrm{em}}$ 和 Stokes 位移不一 致, 连有供电子基的产物的量子产率大于连有吸电子基 的产物, 这与环上电子云密度的大小顺序是一致的. 产 物 $\mathbf{4 z}$ 的量子产率最大 $(0.960)$, 说明稠合环数对量子产 率有较大影响, 稠合环数越多, 共轭体系越长, 离域 $\pi$ 电子书越多, 产物量子产率越大.

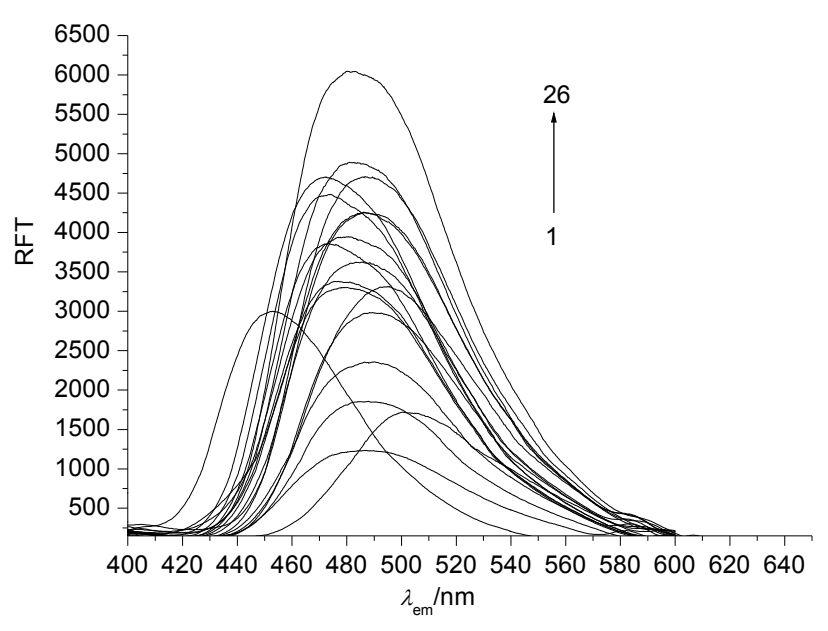

图 4 产物 4 的荧光发射光谱

Figure 4 Fluorescence emission spectra of the product 4

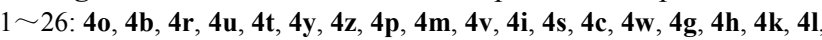
$4 x, 4 j, 4 n, 4 f, 4 q, 4 d, 4 e, 4 a$

表 1 化合物 4 的发光性质

Table 1 Luminescence properties of compound 4

\begin{tabular}{|c|c|c|c|c|c|c|c|c|}
\hline 化合物 & $\mathrm{R}^{1}$ & $\mathrm{R}^{2}$ & $E_{\max (\mathrm{abs})} /\left(\mathrm{L} \cdot \mathrm{mol}^{-1} \cdot \mathrm{cm}^{-1}\right)$ & $\lambda_{\mathrm{abs}} / \mathrm{nm}$ & $\lambda_{\mathrm{em}} / \mathrm{nm}$ & $\mathrm{RFI}^{a}$ & Stoke/nm & $\Phi^{b}$ \\
\hline 9,10-二苯蒽 & & & 2.221 & 261 & 408 & 9989 & 147 & 0.660 \\
\hline $4 a$ & $\mathrm{C}_{6} \mathrm{H}_{5}$ & $\mathrm{H}$ & 3.067 & 230 & 482 & 6064 & 197 & 0.707 \\
\hline $4 b$ & $4-\mathrm{CN}$ & $\mathrm{H}$ & 3.882 & 223 & 501 & 1720 & 203 & 0.268 \\
\hline $4 c$ & $4-\mathrm{CH}_{3}$ & $\mathrm{H}$ & 3.776 & 221 & 477 & 3380 & 178 & 0.513 \\
\hline 4d & $4-\mathrm{OH}$ & $\mathrm{H}$ & 3.036 & 229 & 471 & 4747 & 172 & 0.550 \\
\hline $4 e$ & 2-OMe & $\mathrm{H}$ & 3.098 & 231 & 481 & 4891 & 189 & 0.558 \\
\hline $4 f$ & 4-OMe & $\mathrm{H}$ & 3.004 & 228 & 474 & 4485 & 175 & 0.514 \\
\hline $4 \mathrm{~g}$ & $2,3-(\mathrm{OMe})_{2}$ & $\mathrm{H}$ & 2.927 & 229 & 485 & 3624 & 193 & 0.404 \\
\hline $4 h$ & $3,4-(\mathrm{OMe})_{2}$ & $\mathrm{H}$ & 3.125 & 230 & 473 & 3859 & 177 & 0.460 \\
\hline $4 i$ & $3,4,5-(\mathrm{OMe})_{3}$ & $\mathrm{H}$ & 3.051 & 229 & 480 & 3302 & 181 & 0.384 \\
\hline $4 j$ & $3-\mathrm{F}$ & $\mathrm{H}$ & 4.087 & 222 & 486 & 4254 & 191 & 0.699 \\
\hline $4 k$ & $4-\mathrm{F}$ & $\mathrm{H}$ & 3.036 & 231 & 481 & 3946 & 186 & 0.457 \\
\hline 41 & $2-\mathrm{Cl}$ & $\mathrm{H}$ & 2.880 & 229 & 490 & 3946 & 199 & 0.259 \\
\hline $4 m$ & $3-\mathrm{Cl}$ & $\mathrm{H}$ & 4.012 & 224 & 453 & 3000 & 155 & 0.493 \\
\hline $4 n$ & $4-\mathrm{Cl}$ & $\mathrm{H}$ & 2.989 & 222 & 486 & 4256 & 186 & 0.485 \\
\hline 40 & $2-\mathrm{Br}$ & $\mathrm{H}$ & 3.051 & 228 & 487 & 1234 & 197 & 0.144 \\
\hline $4 p$ & $3-\mathrm{Br}$ & $\mathrm{H}$ & 2.973 & 229 & 489 & 2984 & 193 & 0.338 \\
\hline $4 q$ & $4-\mathrm{Br}$ & $\mathrm{H}$ & 3.004 & 229 & 487 & 4710 & 188 & 0.540 \\
\hline $4 r$ & 4-I & $\mathrm{H}$ & 4.087 & 223 & 488 & 1857 & 185 & 0.305 \\
\hline $4 s$ & $2,4-\mathrm{Cl}_{2}$ & $\mathrm{H}$ & 3.160 & 233 & 495 & 3315 & 203 & 0.399 \\
\hline $4 t$ & 4-OMe & $4-F$ & 5.570 & 240 & 472 & 2007 & 170 & 0.426 \\
\hline $4 u$ & 4-OMe & $4-\mathrm{Cl}$ & 5.796 & 240 & 485 & 1916 & 181 & 0.424 \\
\hline $4 v$ & 4-OMe & $4-\mathrm{OH}$ & 4.553 & 219 & 465 & 3088 & 155 & 0.536 \\
\hline $4 w$ & 4-OMe & 4-OMe & 4.481 & 218 & 465 & 3410 & 155 & 0.583 \\
\hline $4 x$ & 4-OMe & $5-\mathrm{CH}_{3}$ & 4.592 & 219 & 488 & 4087 & 184 & 0.716 \\
\hline $4 y$ & 4-OMe & $5-\mathrm{OMe}$ & 5.656 & 237 & 477 & 2867 & 176 & 0.618 \\
\hline $4 z$ & 4-OMe & & 9.999 & 240 & 460 & 2928 & 168 & 0.96 \\
\hline
\end{tabular}

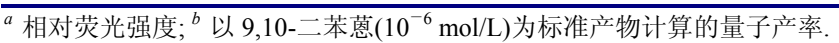


综上所述, 取代基电子云密度是影响产物 4 荧光性 能的主要因素: $R^{1}$ 和 $R^{2}$ 具有相反的电子效应且差别越 大, 电子流动有方向性和驱动力越强, 分子的极性越大, 稳定性就越高, 产物荧光发射波长越长、量子产率越大、 Stokes 位移越大; $R^{1}$ 和 $R^{2}$ 具有相同电子效应时, $R^{1}$ 为吸 电子基的产物的荧光发射波长和 Stokes 位移大于 $\mathrm{R}^{1}$ 为 供电子基的产物, 连有供电子基的产物的量子产率大于 连有吸电子基的产物.

\section{2 化合物 6 的苂光性能构效关系}

我们以同样的方法测定了化合物 $\mathbf{6}$ 的荧光性质. 由 表 2 和图 5 可以看出:

(1)产物的发射波长均在可见光范围内 $\left(\lambda_{\mathrm{em}}=408 \sim\right.$ $509 \mathrm{~nm}$ ). 发射波长的大小与两个取代基的电子效应有 关: 除了个别产物外, 连有吸电子基的芳香醛得到的产 物的荧光发射波长大于连有供电子基的芳香醛得到的 产物.

(2)产物的 Stokes 位移均较大 ( $95 \sim 203 \mathrm{~nm})$.

(3)所有产物的激发波长或发射波长都在一定的变 化范围内，说明其发光性质主要来自于其骨架，但取代 基的电子效应、空间位阻对发射波长和激发波长产生一 定的影响，导致它们产生一定程度的红移或蓝移.

(4)产物的荧光量子产率为 $0.110 \sim 0.780$, 其中化合 物 $6 a$ 的量子产率超过了参比物, 具有进一步研究的价 值.

(5)与化合物 4 相比，对于取代基相同的苯甲醛，化
合物 6 的苂光量子产率较相似，这可能是因为两个骨架 差别不大, 氧原子和氮原子的原子结构相差不大. 产物 $\mathbf{6}$ 的苂光性能构效关系与产物 $\mathbf{4}$ 相似.

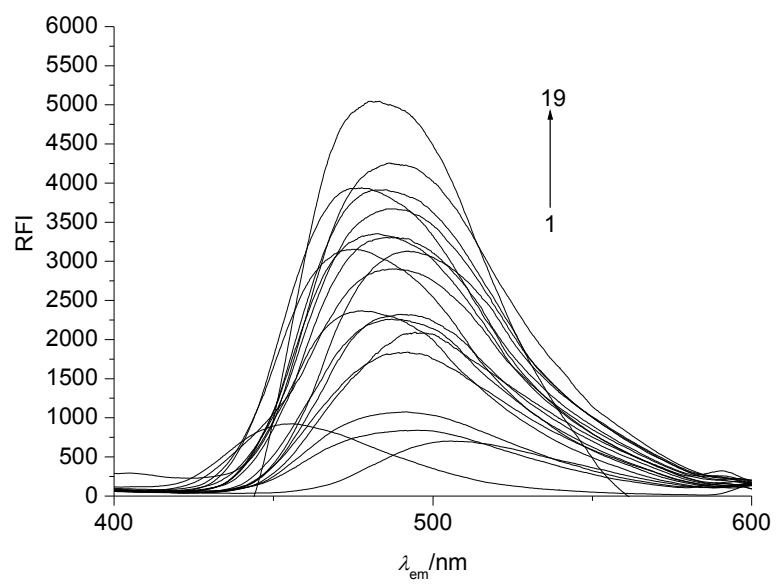

图 5 产物 $\mathbf{6}$ 的菼光发射光谱

Figure 5 Fluorescence emission spectra of the product 6 $1 \sim 19:$ 6a, 6n, 6f, 6k, 6g, 6c, 6e, 6d, 6p, 6j, 6h, 6q, 6r, 6s, 6o, 6m, 6i, 6b 6l

\section{3 化合物 4 对金属离子的识别}

为进一步验证上述构效关系，探索产物配位能力， 开发其在配位化学、金属离子识别等领域的应用，我们 测定了金属离子对产物 4 苂光性质的影响，初步探索了 产物 4 对一些过渡和稀土金属离子的识别.

分别移取 $1 \mathrm{~mL}$ 产物 4 (无水乙醇溶液, $10^{-5} \mathrm{~mol} / \mathrm{L}$ )

表 2 化合物 6 的发光性质

Table 2 The luminescence properties of compound 6

\begin{tabular}{|c|c|c|c|c|c|c|c|}
\hline 化合物 & $\mathrm{R}$ & $E_{\max }(\mathrm{abs}) /\left(\mathrm{mol} \cdot \mathrm{L}^{-1} \cdot \mathrm{cm}^{-1}\right)$ & $\lambda_{\mathrm{abs}} / \mathrm{nm}$ & $\lambda_{\mathrm{em}} / \mathrm{nm}$ & $\mathrm{RFI}^{a}$ & Stokes/nm & $\Phi^{b}$ \\
\hline 9,10-二苯蒽 & & 2.221 & 261 & 408 & 9989 & 147 & 0.660 \\
\hline $6 a$ & $\mathrm{H}$ & 4.067 & 219 & 482 & 5046 & 197 & 0.780 \\
\hline 6b & $4-\mathrm{CN}$ & 4.097 & 219 & 509 & 703 & 205 & 0.110 \\
\hline $6 c$ & $4-\mathrm{CH}_{3}$ & 4.089 & 216 & 482 & 3353 & 182 & 0.523 \\
\hline 6d & $4-\mathrm{OH}$ & 4.056 & 220 & 476 & 3154 & 176 & 0.488 \\
\hline $6 e$ & $2-\mathrm{MeO}$ & 4.080 & 219 & 486 & 3304 & 194 & 0.514 \\
\hline 6f & $4-\mathrm{MeO}$ & 4.072 & 220 & 478 & 3940 & 177 & 0.612 \\
\hline $6 \mathrm{~g}$ & $2,3-(\mathrm{MeO})_{2}$ & 4.085 & 219 & 487 & 3671 & 192 & 0.572 \\
\hline $6 h$ & $3,4-(\mathrm{MeO})_{2}$ & 4.087 & 219 & 478 & 2368 & 179 & 0.370 \\
\hline $6 \mathbf{i}$ & $3,4,5-(\mathrm{MeO})_{3}$ & 4.082 & 217 & 491 & 839 & 190 & 0.130 \\
\hline $6 \mathbf{j}$ & $3-\mathrm{F}$ & 4.084 & 219 & 488 & 2903 & 190 & 0.452 \\
\hline $6 \mathbf{k}$ & $4-\mathrm{F}$ & 4.089 & 222 & 485 & 3915 & 187 & 0.610 \\
\hline 61 & $2-\mathrm{Cl}$ & 4.056 & 218 & 490 & 688 & 185 & 0.106 \\
\hline $6 \mathrm{~m}$ & $3-\mathrm{Cl}$ & 4.764 & 219 & 455 & 923 & 157 & 0.168 \\
\hline $6 n$ & $4-\mathrm{Cl}$ & 2.989 & 222 & 486 & 4256 & 186 & 0.485 \\
\hline 60 & $2-\mathrm{Br}$ & 4.068 & 216 & 491 & 1076 & 103 & 0.167 \\
\hline $6 p$ & $3-\mathrm{Br}$ & 4.065 & 220 & 492 & 131 & 95 & 0.485 \\
\hline $6 q$ & $4-\mathrm{Br}$ & 4.109 & 218.5 & 490 & 32 & 102 & 0.364 \\
\hline $6 r$ & 4-I & 4.077 & 219 & 490 & 257 & 186 & 0.351 \\
\hline $6 s$ & $2,4-\mathrm{Cl}_{2}$ & 4.087 & 219 & 497 & 2084 & 202 & 0.325 \\
\hline
\end{tabular}

相对苂光强度; ${ }^{b}$ 以 9,10 -二苯葱 $\left(10^{-6} \mathrm{~mol} / \mathrm{L}\right)$ 为标准产物计算的量子产率. 
和金属离子(水溶液, $10^{-5} \mathrm{~mol} / \mathrm{L}$ )于一系列 $10 \mathrm{~mL}$ 容量瓶 中, 用无水乙醇稀释至刻度, $60{ }^{\circ} \mathrm{C}$ 分别测量其荧光强 度, 结果见表 3 和图 6.

图 6 为化合物 $4 \mathrm{a}$ 在 $60{ }^{\circ} \mathrm{C}$ 时, 9 种离子对其荧光发 射光谱的影响. 曲线 1 表示 $60{ }^{\circ} \mathrm{C}$ 时 $4 \mathbf{a}$ 的苂光谱线图, 曲线 2 10 分别表示在 $60{ }^{\circ} \mathrm{C}$ 时 $\mathrm{Y}^{3+}, \mathrm{Au}^{4+}, \mathrm{Fe}^{3+}, \mathrm{Co}^{2+}$, $\mathrm{Sn}^{2+}, \mathrm{In}^{3+}, \mathrm{Cu}^{2+}, \mathrm{Mn}^{2+}, \mathrm{Zn}^{2+}$ 存在下 $4 \mathbf{a}$ 的苂光谱线图.

从表 3 可以看出所选 9 种离子对部分产物的影响情 况不一致, 对于同一个化合物, 不同的金属离子对其荧 光相对强度的影响不一致, 有的增强了其荧光有的则使 其荧光淬灭, 不管是荧光增强还是荧光淬灭, 都可以作 为测定这些金属离子的方法.

各金属离子对 $4 \mathrm{a}, 4 \mathrm{~h}, 4 \mathrm{n}$ 的影响比较明显且均呈增 强荧光的趋势, 荧光增强的幅度也比较大, 说明这几个 化合物是各金属离子比较理想的探针. 从结构上看, 金 属离子对这 3 个化合物的荧光增强没有明显规律.

将与金属离子反应后荧光强度变化最大者标出(加 粗)可以看出, $4 \mathrm{e}$ 和 $4 \mathrm{n}$ 均能与 3 种离子反应发生最大荧 光强度变化. 所有化合物和离子反应中, 苂光强度变化 最大的是 $4 \mathrm{~h}$ 与 $\mathrm{Zn}^{2+}$ 的反应, 说明 $4 \mathrm{~h}$ 是非常优良的 $\mathrm{Zn}^{2+}$ 苂光探针, 本实验室将做进一步研究.

分离子讨论, 对 $\mathrm{Zn}^{2+}$ 的识别能力最强的是化合物 $4 \mathrm{~h}$; 对 $\mathrm{Cu}^{2+}$ 的识别能力最强的是化合物 $4 \mathrm{~s}$; 对 $\mathrm{Mn}^{2+}$ 的 识别能力最强的是化合物 $4 \mathrm{n}$; 对 $\mathrm{Sn}^{2+}$ 的识别能力最强 的是化合物 $4 \mathrm{n}$; 对 $\mathrm{Co}^{2+}$ 的识别能力最强的是化合物 $4 \mathrm{e}$; 对 $\mathrm{Fe}^{3+}$ 的识别能力最强的是化合物 $4 \mathrm{n}$; 对 $\mathrm{In}^{3+}$ 的识别能 力最强的是化合物 $4 \mathrm{~h}$; 对 $\mathrm{Y}^{3+}$ 的识别能力最强的是化合 $4 \mathrm{e}$; 对 $\mathrm{Au}^{4+}$ 的识别能力最强的是化合物 $4 \mathrm{e}$, 相互作用最 强的是 $4 \mathbf{h}$ 与 $\mathrm{Zn}^{2+}$.

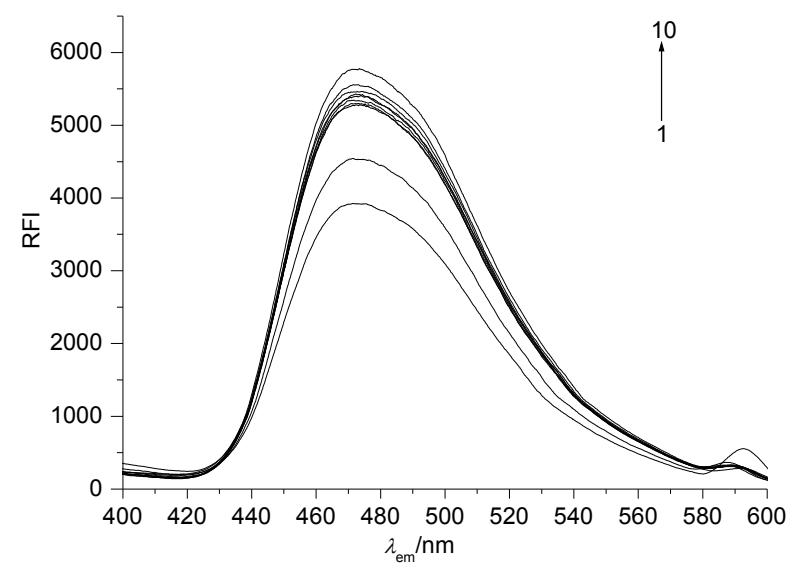

图 6 化合物 $4 a$ 对 9 种金属离子的识别

Figure 6 Identification of compound $4 \mathbf{a}$ to nine metal ions $1 \sim 10: 4 a, \mathrm{Y}^{3+}, \mathrm{Au}^{4+}, \mathrm{Fe}^{3+}, \mathrm{Co}^{2+}, \mathrm{Sn}^{2+}, \mathrm{In}^{3+}, \mathrm{Cu}^{2+}, \mathrm{Zn}^{2+}, \mathrm{Mn}^{2+}$

图 6 为化合物 $4 \mathrm{a}$ 在 $60{ }^{\circ} \mathrm{C}$ 时, 9 种离子对其荧光发 射光谱的影响. 曲线 1 表示 $60{ }^{\circ} \mathrm{C}$ 时 $\mathbf{4 a}$ 的荧光谱线图, 曲线 2 10 分别表示在 $60{ }^{\circ} \mathrm{C}$ 时 $\mathrm{Y}^{3+}, \mathrm{Au}^{4+}, \mathrm{Fe}^{3+}, \mathrm{Co}^{2+}$, $\mathrm{Sn}^{2+}, \mathrm{In}^{3+}, \mathrm{Cu}^{2+}, \mathrm{Zn}^{2+}, \mathrm{Mn}^{2+}$ 存在下 $4 \mathrm{a}$ 的荧光谱线图.

\section{2 结论}

本论文对含二氮杂菜砌块化合物的荧光性质构效 关系进行了研究. 结果表明: 所有产物均发射可裸眼识 别的强烈荧光，其荧光发射波长均在可见区，显示了该 类化合物强大发光性能. 分子中具有多个配位点, 为进 一步研究其在配位化学、发光材料、荧光探针等方面的 应用打下了坚实的基础.

综上所述，该类化合物具有开发为发光材料或荧光 探针的潜力. 本研究为其在荧光发光材料以及苂光衍生 化试剂方面的开发和应用打下了基础.

表 3 化合物 4 对 9 种金属离子的荧光识别 $(\Delta F)^{a}$

Table 3 Fluorescence identification of compound 4 to 9 kinds of metal ions $(\Delta F)$

\begin{tabular}{crrrrrrrrrr}
\hline 化合物 & $\mathrm{IRF}$ & $\mathrm{Zn}^{2+}$ & $\mathrm{Cu}^{2+}$ & $\mathrm{Mn}^{2+}$ & $\mathrm{Sn}^{2+}$ & $\mathrm{Co}^{2+}$ & $\mathrm{Fe}^{3+}$ & $\mathrm{In}^{3+}$ & $\mathrm{Y}^{3+}$ & $\mathrm{Au}^{4+}$ \\
\hline $\mathbf{4 a}$ & 6695 & 1227 & 1149 & 1475 & 779 & 749 & 461 & 1145 & 62 & 102 \\
$\mathbf{4 d}$ & 6195 & 118 & 312 & 417 & -822 & -1922 & -902 & -1298 & -707 & -744 \\
$\mathbf{4 e}$ & 5618 & 1368 & 1401 & 913 & 1865 & $\mathbf{1 9 8 5}$ & 1727 & -801 & $\mathbf{2 6 1 0}$ & $\mathbf{2 3 2 0}$ \\
$\mathbf{4 f}$ & 5787 & 677 & 799 & 763 & 100 & 112 & 943 & -8 & 817 & 951 \\
$\mathbf{4 g}$ & 5665 & 734 & 497 & -85 & 1283 & 1055 & 463 & 323 & 508 & 528 \\
$\mathbf{4 h}$ & 3923 & $\mathbf{2 7 3 7}$ & 1357 & 1377 & 1487 & 1420 & 1506 & $\mathbf{1 8 5 4}$ & 1542 & 1635 \\
$\mathbf{4 i}$ & 4144 & 679 & -88 & 962 & 130 & 713 & 301 & -340 & 692 & 1234 \\
$\mathbf{4 k}$ & 5536 & 1798 & 493 & 500 & -35 & 226 & 203 & -114 & -372 & 180 \\
$\mathbf{4 l}$ & 5192 & 1570 & -263 & 202 & -400 & -145 & 405 & -221 & -339 & -40 \\
$\mathbf{4 n}$ & 5156 & 1335 & 1658 & $\mathbf{1 9 8 3}$ & $\mathbf{1 9 6 2}$ & 1696 & $\mathbf{1 8 0 6}$ & 1821 & 1898 & 2064 \\
$\mathbf{4 0}$ & 1552 & -27 & 11 & 231 & -15 & -4 & -213 & -165 & -245 & -48 \\
$\mathbf{4 p}$ & 3938 & -719 & -305 & -303 & -617 & -155 & -702 & -131 & 120 & -621 \\
$\mathbf{4 q}$ & 5637 & -375 & 1217 & -234 & -386 & -372 & 465 & -267 & -1459 & -128 \\
$\mathbf{4 s}$ & 3005 & -1010 & $\mathbf{2 2 0 2}$ & 1071 & 1169 & 794 & 1578 & 1096 & 818 & 1297 \\
\hline
\end{tabular}

“没有标注 “负号” 的表示相对苂光强度 $F$ 的增加值, 标注 “负号” 的表示 $F$ 的减小值. 


\section{3 实验部分}

\section{1 仪器与试剂}

主要仪器: FTIR-Tensor-27 型红外光谱仪 $(\mathrm{KBr}$ 压 片), Bruker Avance DPX-400 MHz 型核共振仪(TMS 为 内标), Bruker MicrOTOF-QII 型质谱仪, F-4500 苂光分 光光度计 (日本日立)、恒温支架、比色皿 $(1 \mathrm{~cm} \times 1 \mathrm{~cm} \times 4$ $\mathrm{cm}) 、$ 狭缝宽度为 5 和 $10 \mathrm{~nm}, \mathrm{UV}-2500 \mathrm{PC}$ 紫外可见分光 光谱仪(岛津).

主要试剂：邻着基苯乙酮(A.R., 上海试剂三厂), 薄层层析硅胶(C. P., 青岛市基亿达硅胶试剂厂), 水为 去离子水, 其余均为国产分析纯.

化合物均配制成浓度均为 $1.0 \times 10^{-6} \mathrm{~mol} / \mathrm{L}$ 的无水 乙醇溶液，室温下测其最大紫外吸收波长和苂光性质.

\section{2 实验步骤}

\section{2 .1 化合物 4 的合成}

在干燥的 $100 \mathrm{~mL}$ 三颈烧瓶中, 依次加入丙二腈(1) $(2 \mathrm{mmol}) 、$ 芳醛 $(2)(1 \mathrm{mmol}) 、$ 邻羊基苯乙酮 $(3)(1 \mathrm{mmol}) 、$ $\mathrm{H}_{2} \mathrm{O}(8 \mathrm{~mL})$ 、乙醇 $(2 \mathrm{~mL})$ 和硅胶 $(0.03 \mathrm{~g}), 80{ }^{\circ} \mathrm{C}$ 条件下加 热反应(TLC 跟踪检测). 反应结束后, 冷却体系, 真空 抽滤得粗产品, 用冷乙醇洗涤几次, DMF 加热溶解, 趁 热抽滤除去硅胶, DMF 重结晶得到产物 4 .

\section{2 .2 化合物 6 的合成}

在干燥的 $100 \mathrm{~mL}$ 烧瓶中, 加入邻氨基苯乙酮 5 (1 $\mathrm{mmol}) 、$ 芳醛 2 (1 mmol)、丙二腈 $\mathbf{1}(2 \mathrm{mmol}) 、 8 \mathrm{~mL} \mathrm{H} \mathrm{H}_{2} \mathrm{O}$ 和 $2 \mathrm{~mL}$ 无水乙醇, 最后加入催化剂硅胶 $0.03 \mathrm{~g}, 80{ }^{\circ} \mathrm{C}$ 条 件下回流反应(TLC 跟踪检测). 反应结束后, 冷却体系, 真空抽滤得粗产品, 用冷乙醇洗涤几次, DMF 加热溶解, 趁热抽滤除去硅胶, 重结晶, 抽滤收集滤液, 用石油醚乙酸乙酯 $(V: V=2: 1)$ 柱层析得产物 6 .

\subsection{3 化合物 4 和 $\mathbf{6}$ 的表征数据}

化合物 4 和 6 的物理性质和表征数据与参考文献 [27]一致。

\section{References}

[1] (a) Chen, G.-Z. Fluorescence Analysis, 3rd ed., Science Press, Beijing, 2006 (in Chinese).

(陈国珍，荧光分析法(第三版)，科学出版社，北京，2006.)

(b) Yang, S.-X.; Li, Y.-T.; Wang, Y.-L. Modern Instrumental Analysis Tutorial, Chemical Industry Press, Beijing, 2009 (in Chinese).

(杨守祥, 李燕婷, 王宜伦, 现代仪器分析教程, 化学工业出版 社, 北京, 2009.)

[2] Leonid, G. V.; Ilya, V. V.; Tatyana, N. B.; Alexey, V. V. J. Org. Chem. 2008, 73, 4596.

[3] Gilis, P. M.; Haemers, A.; Bollaert, W. J. Heterocycl. Chem. 1980,
$17,717$.

[4] Marchese, A.; Debbia, E. A.; Schito, G. C. J. Antimicrob. Chemother. 2000, 46, 11 .

[5] Zeng, L.-F.; Wang, Y.; Kazemi, R.; Xu, S.-L.; Xu, Z.-L.; Sanchez, T. W.; Yang, L.-M.; Debnath, B.; Odde, S.; Xie, H.; Zheng, Y.-T.; Ding, J.; Neamati, N.; Long, Y.-Q. J. Med. Chem. 2012, 55, 9492.

[6] Antoine, H.; Sabrina, B.; Véronique, L.; Michel, B.; Aline, D.; François, D.; Ronan, B.; Bruno, P.; Pierre, R.; Sylvain, R. J. Med. Chem. 2003, 46, 138.

[7] Vivek, K.; Alka, M.; Vinod, K. S.; Manupriya, V.; Narendra, J.; Anu, T. S.; Manu, J.; Pramod, K. S.; Raghuveer, I.; Anand, C. B. J Enzyme Inhib. Med. Chem. 2009, 24, 1169.

[8] Evgeny, K.; Thomas, S. D.; Yves, P.; Mark, C. J. Med. Chem. 2010 53,8716 .

[9] Andrew, M. T.; Cleo, J. C. C.; James, M. H.; Stacey, B.; Brian, G H.; Aneesa, M. A.; Alan, J. K.; Denise, L. D.; Randall, W. S.; Sandra, J. P.; Patrick, W. V.; Bill, J. R.; William, L. E.; Wayne, K.; Wilbur, R. L.; Hollis, S.; William, A. D. J. Med. Chem. 2000, 43, 4200.

[10] Wang, Y.; Xu, Z.-L.; Ai, J.; Peng, X.; Lin, J.-P.; Ji, Y.-C.; Geng, M.-Y.; Long, Y.-Q. Org. Biomol. Chem. 2013, 11, 1545.

[11] Falardeau, G.; Lachance, H.; St-Pierre, A.; Yannopoulos, C. G.; Drouin, M.; Bédard, J.; Chan. L. Bioorg. Med. Chem. Lett. 2005, $15,1693$.

[12] Thompson, A. M.; Delaney, A. M.; Hamby, J. M.; Schroeder, M. C.; Spoon, T. A.; Crean, S. M.; Showalter, H. D. H.; Denny, W. A. J. Med. Chem. 2005, 48, 4628.

[13] Kuar, M.; Bahia, M. S.; Silakari, O. J. Chem. Inf. Model. 2012, 52, 2619.

[14] Liu, C.-X.; Tang, X.-L.; Li, P.-L.; Li, G.-Q. Org. Lett. 2012, 14, 1994.

[15] Deady, L. W.; Rodemann, T.; Zhuang, L.; Baguley, B. C.; Denny, W. A. J. Med. Chem. 2003, 46, 1049.

[16] Lirvinov, V. P. Adv. Heterocycl. Chem. 2006, 91, 222.

[17] Meanwell, N. A. J. Med. Chem. 2011, 54, 2529.

[18] Stern, E.; Muccioli, G. G.; Bosier, B.; Hamtiaux, L.; Millet, R.; Poupaert, J. H.; Hénichart, J. P.; Depreux, P.; Goossens, J. F.; Lambert, D. M. J. Med. Chem. 2007, 50, 5471.

[19] Helmut, H.; Juergen, P.; Hans, Z.; Bruno, W.; Otto, K. W. DE 3907938, 1990 [Chem. Abstr. 1991, 114, 122342f].

[20] Che, C.-M.; Wan, C.-W.; Ho, K.-Y.; Zhou, Z.-Y. New J. Chem. 2001, 25, 63.

[21] Fu, W.-F.; Jia, L.-F.; Mu, W.-H.; Gan, X.; Zhang, J.-B.; Liu, P.-H.; Cao, Q.-Y.; Zhang, G.-J.; Quan, L.; Lv, X.-J.; Xu, Q.-Q. Inorg. Chem. 2010, 49, 4524.

[22] Wu, Y.-Y.; Chen, Y.; Gou, G.-Z.; Mu, W.-H.; Lv, X.-J.; Du, M.-L.; Fu, W.-F. Org. Lett. 2012, 14, 5226

[23] Fernández-Mato, A.; García, M. D.; Peinador, C.; Quintela, J. M.; Sánchez-Andújar, M.; Pato-Doldán, B.; Señarís-Rodríguez, M. A.; Tordera, D.; Bolink, H. J. Cryst. Growth Des. 2013, 13, 460.

[24] Zink, D. M.; Bächle, M.; Baumann, T.; Nieger, M.; Kühn, M.; Wang, C.; Klopper, W.; Monkowius, U.; Hofbeck, T.; Yersin, H.; Bräse, S. Inorg. Chem., 2013, 52, 2292.

[25] Li, L.; Li, J.-Z.; Wang, H.-Y.; Zhang, H.-M.; Fu, W. Chin. J. Chem. 2012, 30, 1801.

[26] Ravindranathan, K. P.; Mandiyan, V.; Ekkati, A. R.; Bae, J. H.; Schlessinger, J.; Jorgensen, W. L. J. Med. Chem. 2010, 53, 1662.

[27] Wu, H.; Lin, W.; Wan, Y.; Xin, H.-Q.; Shi, D.-Q.; Shi, Y.-H.; Yuan, R.; Bo, R.-C.; Yin, W. J. Comb. Chem. 2010, 12, 31. 